\title{
Impact responses of slender composite plates for bird-strike testing of fan blades
}

\author{
Yadong Zhou ${ }^{\mathrm{a}^{*}}$ (1) \\ Youchao Sun ${ }^{\mathrm{a}}$ \\ Tianlin Huang ${ }^{b}$ (D) \\ a College of Civil Aviation, Nanjing University of Aeronautics and Astronautics, Nanjing 211100, China. E-mail: yzhou@nuaa.edu.cn, \\ sunyc@nuaa.edu.cn \\ b Commercial Aircraft Engine Co., LTD, Aero Engine Cooperation of China, Shanghai 201100, China. E-mail: aecc_htl@163.com \\ *Corresponding author
}

https://doi.org/10.1590/1679-78255809

\begin{abstract}
Bird-strike failure of fan blades is one of the basic challenges for the safety of aircraft engines. Simplified slender blade-like plates are always used to evaluate the impact-induced damage mechanism at design stage. One undesirable issue is the failure at the root of clamped slender plates, which cannot recover the real case of twisted blades. For this purpose, three different strategies were exploited to obtain desirable deformation and stress responses, namely the impact location, additional weight, and the boundary condition. Numerical models of the simplified slender blade and the bird projectile were constructed by using finite element method (FEM) and smoothed particle hydrodynamics (SPH) approaches. The impact deformations and stress distributions were comparatively investigated in detail. The numerical results show that changing the boundary condition is the most effective way to obtain preferable impact responses for further failure analysis of real fan blades. Present results will be useful to future experimental design of simplified bird-strike testing.
\end{abstract}

\section{Key words}

bird strike; impact response; simplified blade; equivalent experiment

\section{Introduction}

Aircraft engines are the critical front-facing components, and the fan blades may be subjected to bird strike with high probability (Abrate, 2016). Capability of ingesting birds weighing up to $1.8 \mathrm{~kg}$ without sustaining damage is required so that no fatal threat will pose to the people in aircraft. Bird strike of engine blades has received more attentions than before. Meguid et al. (Meguid, Mao, \& $\mathrm{Ng}$, 2008) studied the impact behaviour of an artificial bird impinging a flexible aeroengine fan blade. Kim et al. (Kim, Zammit, Siddens, \& Bayandor, 2011) investigated the soft impact damage assessment of composite fan stage assemblies. Sinha et al. (Sinha, Turner, \& Jain, 2011) investigated the dynamic loading on turbofan blades due to bird strike, where rectangular composite panel was adopted for model validation. Vignjevic et al. (Vignjevic, Orłowski, De Vuyst, \& Campbell, 2013) performed a parametric study of bird strike on engine blades. Amoo (Amoo, 2013) conducted a review on the design and structural analysis of jet engine fan blade structures, including the impact dynamics analysis under bird strike. Mishra et al. (Mishra, Ahmed, \& Srinivasan, 2013) investigated a bird strike incident of a military gas turbine engine. Sinha (Sinha, 2014) obtained transient response of a composite rotating airfoil under bird-slicing impact. Zhang et al. (D. Zhang \& Fei, 2016) conducted bird strike analysis of engine fan 
blades made of titanium alloy. Muir et al. (Muir \& Friedmann, 2016) numerically investigated the structural response of bird-damaged fan blades, where realistic fan configuration was considered in numerical simulations. Liu et al. (J. Liu et al., 2018a) carried out bird strike tests on the rotary engine primary compressor under different impact boundary conditions. Zhang et al. (Z. Zhang, Li, \& Zhang, 2018) investigated the impact behaviours of real bird models striking on rotating engine blades from arbitrary attitude angles. Authors (Zhou, Sun, \& Cai, 2019) recently considered the bird-strike failure analysis of rotating laminated disks for future key applications of aeroengine blades.

It should be noted that bird strike of fan blades involves the problem regarding the angle of attack of the inclined airfoil (Mao, Meguid, \& Ng, 2007), which complicates the issue, such as slicing of a bird by fan blades in the direction of the relative velocity. Future propulsion systems will incorporate advanced composites, particularly in the fan assembly, because of their superior strength and stiffness properties compared to metals (Kim et al., 2011). However, when dealing with composite laminates, full-scale complete tests of aeroengine fan blades become infeasible in the design process due to the associated high cost. Cantilever slender plates are an important experimental configuration to examine the impact resistance of to mimic composite fan blades. Significant contributions have been made toward understanding the bird impact behaviours of these simplified plates. Hou et al. (Hou \& Ruiz, 2007) simulated bird strike on composite panels by means of simple laboratory tests to provide data for modelling and design of composite fan blades. For application to bird-strike problem of composite fan blades, Nishikawa et al. (Nishikawa, Hemmi, \& Takeda, 2011) simplified a unidirectional composite plate for discussing the damage characteristics. They found the transition from the global bending mode at low velocity to the local deformation mode at high velocity. Handschuh et al. (Handschuh et al., 2014) developed the simplified test article geometry to realistically simulate a blade leading edge while decreasing fabrication complexity, where the prepared leading edge test article was clamped along one long edge. Liu et al. (2018b) carried out bird impact tests on the composite plates using the gas gun system. They divided whole impact event into 3 stages, i.e., local deformation stage, post-flow impact stage, and bending deflection stage. Such specimen-level experiments can obtain some critical damage mechanism. Roberts et al. (Roberts et al., 2002) verified that simpler tests using smaller ring sections of engine fan cases were adequate when the evaluation of initial impact damage was the primary concern. Rayavarapu (Rayavarapu, 2016) pointed out that validation studies of bird impact damage at specimen and substructure level should be performed, since the experimental tests are expensive and difficult to perform. The soft-body impact process (displacement and strain field histories) and time-dependent energy exchange can be evaluated with the help of carefully-designed panel-level experiments (D. Li, Zhang, Guo, Xie, \& Jiang, 2019).

Although the structural types are different from blades, the simplification practice for windshields or wing sections can provide valuable reference significance. With the help of the structural similarity regarding the component-level experiments for windshields or wing sections, the four-edge clamped plates can be successfully applied. For example, Guida et al. (M. Guida, Marulo, Meo, \& Russo, 2012) adopted an insightful research route "specimen testing - panel experiment/simulation - wing leading edge experiment/simulation". Guida et al. (M. Guida, Sellitto, Marulo, \& Riccio, 2019) presented an experimental-numerical investigation on the impact damage behaviour of simplified square composite plates with embedded shape memory alloys for aeronautical structural component. Mohagheghian et al. (Mohagheghian et al., 2017) performed the laboratory-scale impact experiments using a gas gun apparatus for betterquantified understanding of soft impact damage on laminated glass windows and more effective design of aircraft windshields. Di Caprio et al. (Di Caprio, Cristillo, Saputo, Guida, \& Riccio, 2019) assessed the bird-strike numerical models by a double-checked and hence the two test cases considered procedure: 1) bird strike on the flat square plate; 2) bird strike on the wing leading edge. These results indicate that the most damaged region in bird-strike tests are always the impact contact area. Such fact can largely facilitate the mechanism investigation into the materials, and can bridging from component-level results to structure-level evaluation. The impact location is also critical for wing leading edge under bird strike. Yu et al. (Yu, Xue, Yao, \& Zahran, 2019) proposed an analytical method for determining the critical location that causes the most severe damage under impact due to bird strike. 


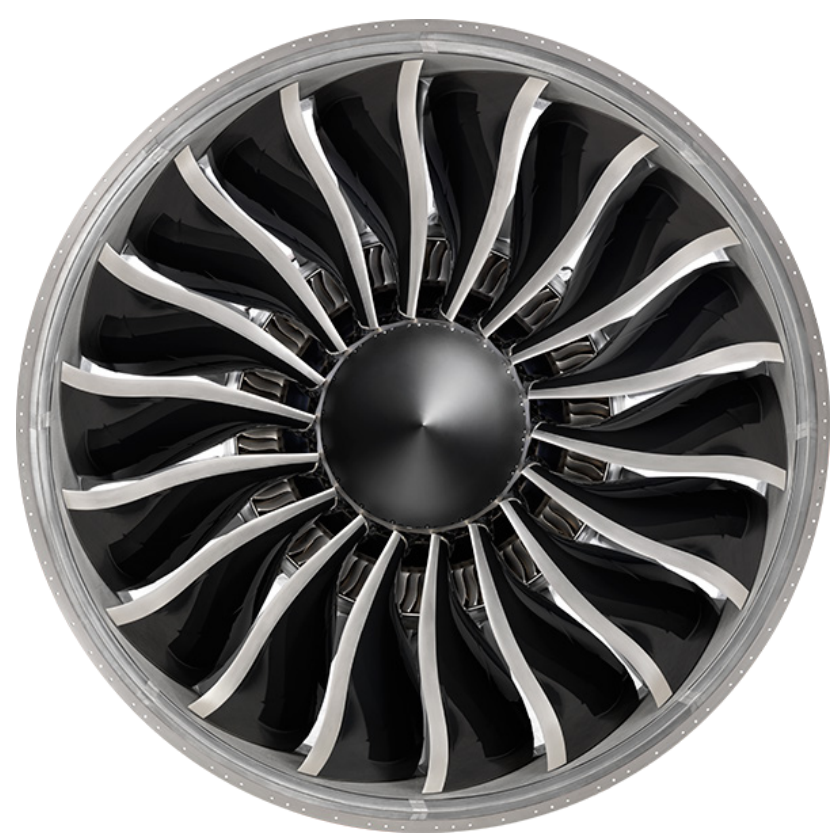

Fig. 1. Carbon-fiber composite fan blades of GEnx engine
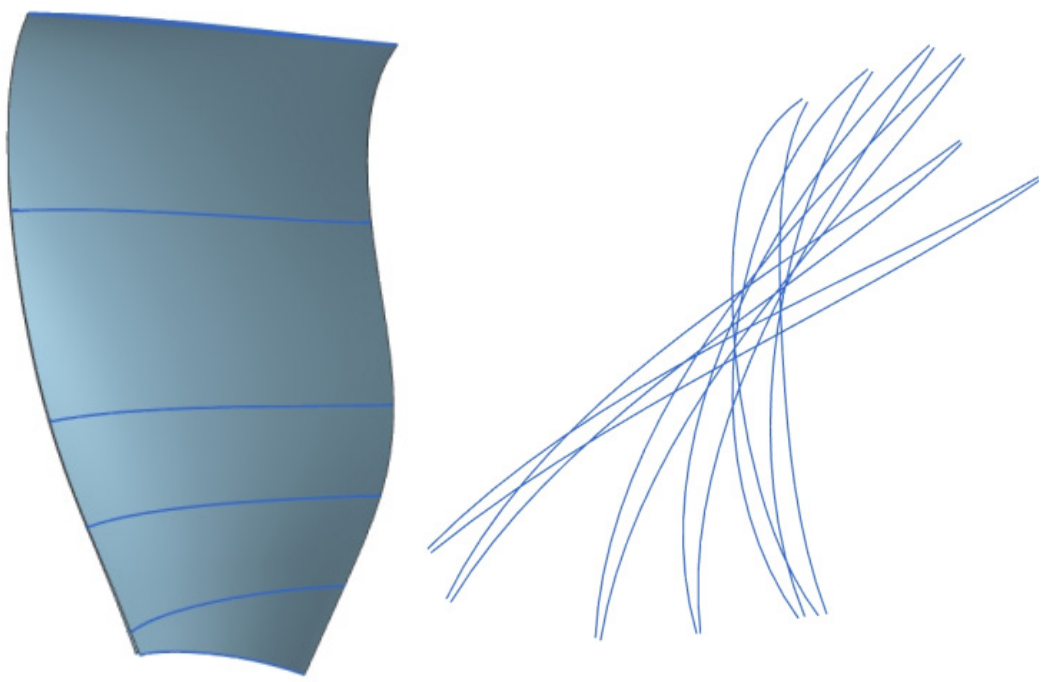

Fig. 2. An example of fan blade airfoil and its profile sections

For the real complex twisted fan blades (e.g. the carbon-fiber composite fan blades of GEnx engine (The GEnx Commercial Aircraft Engine, 2019), as shown in Fig. 1), the most susceptible regions are the impact contact area and the leading edges of the blade (Vignjevic et al., 2013), which slice the bird projectile. From the geometrical profile of the blade, the "stagger angle $\phi(s)$ " at the impact location $s$ due to pretwist in the airfoil can be expressed as $\phi(s)=\phi_{0}+\phi^{\prime} s$ (Sinha et al., 2011). An example of fan blade airfoil and its profile sections are as shown in Fig. 2. The impact-induced peak strain was observed to locate at the leading edge of the blade (Teichman \& Tadros, 1991). The impact region experiences the maximum Hugoniot pressure $\left(p_{H}\right)$, which is evaluated by $p_{H}=\rho_{0} V_{0} V_{s}$, where $\rho_{0}$ is the initial density, $V_{0}$ is the initial velocity and $V_{s}$ is the velocity of shock wave. Vignjevic et al. (Vignjevic et al., 2013) presented that there was a strong influence of bird impact location on the extent of blade deformations. In terms of simplified blade-like components in experiments, however, the maximum impact damages are always located at the root of cantilever slender plates (L. Liu et al., 2018b), which is determined by the characteristic of the structure and loading types. The flat slender plates can be considered as beam-type structures, and hence the bending moments achieve the maximum at the clamped end under bending deformations. Therefore, the similarity between the simplified testing and real operating situation should be intensively considered. Li et al. (Y. Li, Zhang, \& Xue, 2008) studied the similarity law of bird strike for purpose of the small-scale experiment to replace a full-scale one. Mazzariol and Alves (Mazzariol \& Alves, 2019a, 2019b) 
recently developed comprehensive framework of similarity laws for relating scaled models to prototypes under impact loads.

Within such scenario, the aim of present study is to obtain some simulation results to help future experimental workable and useful. The main strategy is to consider different impact locations, to adopt a concentrated mass, and to change the boundary conditions. By comparing the displacement and stress responses of the slender composite plates under bird strike impact, the final goal is to obtain the stress distributions of the simplified flat blade-like plates similar with those in the real fan blades, rather than the undesirable failure at the roots.

\section{Simulations}

The cylindrical projectile was widely used to simulate the bird body (Michele Guida, Marulo, Polito, Meo, \& Riccio, 2009; Heimbs, 2011), with the length-to-diameter ratio of 2 . The geometric and mass details for present simulations are listed in Table 1. The smoothed particle hydrodynamics (SPH) method is applied to describe the bird fluidic behaviour at high velocities. The laminated blades are modelled by finite element method (FEM), with a total of 1809 linear quadrilateral elements of type S4R used in ABAQUS software. The composite ply was modelled as a homogeneous orthotropic elastic material. The plane-stress formulation was adopted and the in-plane stress components are $\sigma=\left[\sigma_{11}\right.$, $\left.\sigma_{22}, \sigma_{12}\right]^{\top}$. A total of 2220 linear hexahedral elements of type C3D8R were created firstly, and then these elements were converted to SPH particles as the explicit dynamic analysis began. The SPH-FEM model is as shown in Fig. 3. The initial velocity of the bird projectile was $120 \mathrm{~m} / \mathrm{s}$.

Table 1. Geometric and mass details of the plate and bird

\begin{tabular}{cc}
\hline Properties & Value \\
\hline Plate length $(\mathrm{mm})$ & 500 \\
Plate width $(\mathrm{mm})$ & 200 \\
Plate thickness $(\mathrm{mm})$ & 24 \\
Plate weight $(\mathrm{kg})$ & 3.84 \\
Bird length $(\mathrm{mm})$ & 200 \\
Bird diameter $(\mathrm{mm})$ & 100 \\
Bird weight $(\mathrm{kg})$ & 1.49 \\
\hline
\end{tabular}

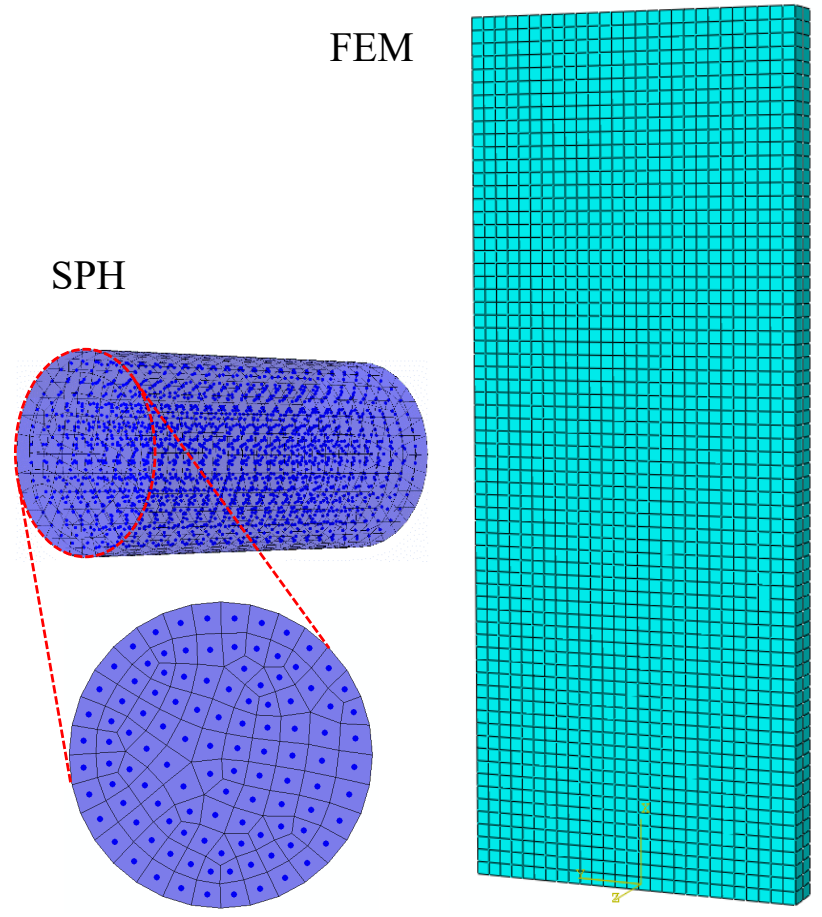

Fig. 3. SPH-FEM model for bird-strike simulation 
The stacking sequence of the unidirectional lamina is as shown in Fig. 4. A total of 16 layers was used. The $0^{\circ}$ direction (longitudinal direction) is along the blade span.

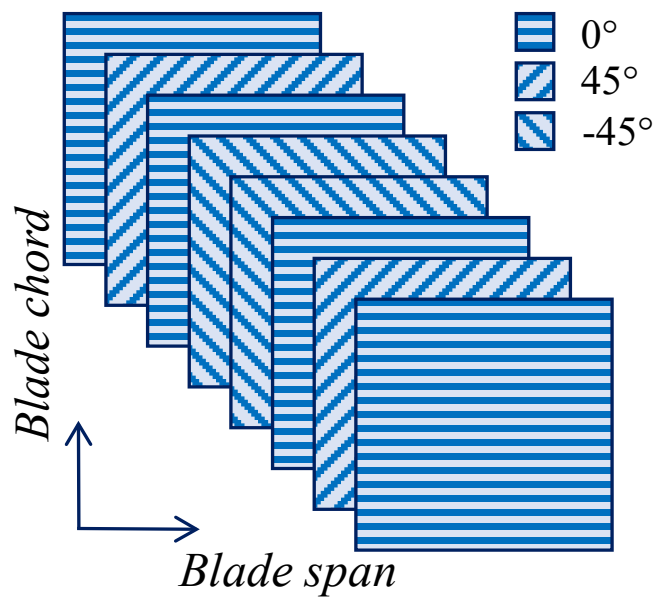

Fig. 4. Stacking sequence of the composite plate (with the symmetric half displayed)

Firstly, the different impact locations were considered, as illustrated in Fig. 5. Among the four locations, 30\%, 50\% and $90 \%$ heights of the blade span are distributed on the axis of symmetry, while the $70 \%$ height of span is eccentrically located, which is for purpose of torsional effect on the impact responses.

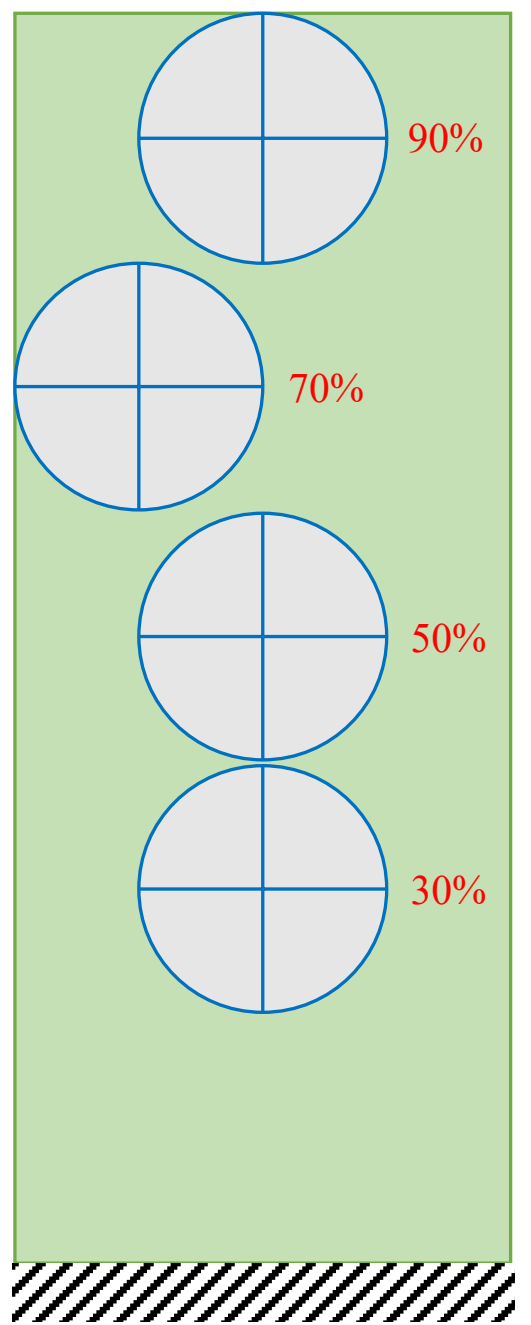

Fig. 5. Different impact locations on the blade 
Fig. 6 show the deformation series of the $50 \%$ height case, i.e. impacted on the geometric center of the plate. It can be seen that the plate behaved as the simply-supported middle-loaded beam at the beginning ( 1 and 2 ms); and then the deformations became globally, and the blade tip experienced large deflection. Because of the symmetry of both the loading and structure, the plate deformed symmetrically in the impact process.
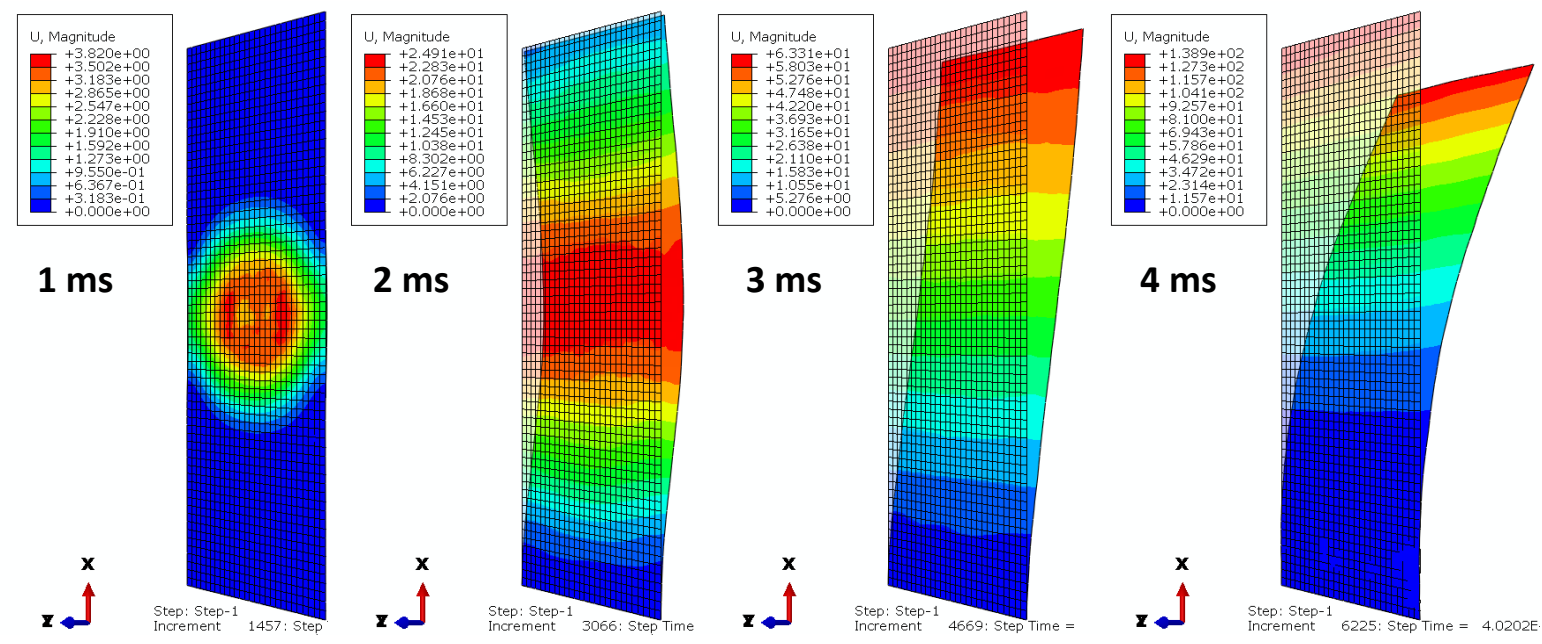

Fig. 6. Deformation series of the $50 \%$ height case

Fig. 7 gives the deformation series of the $90 \%$ height case, i.e. impacted on the location near the blade tip. It can be seen that the plate behaved as the cantilever beam in the whole process. The deflections are also symmetric.
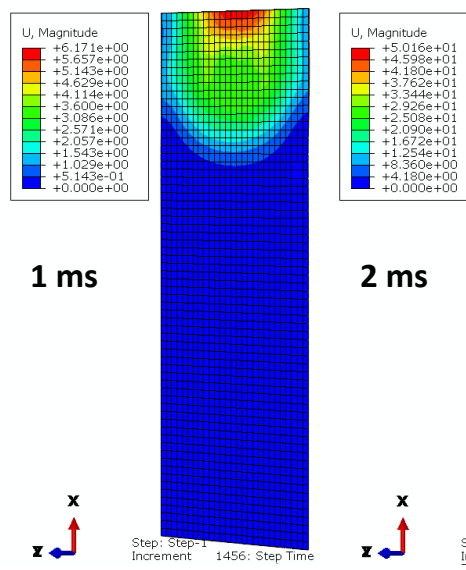
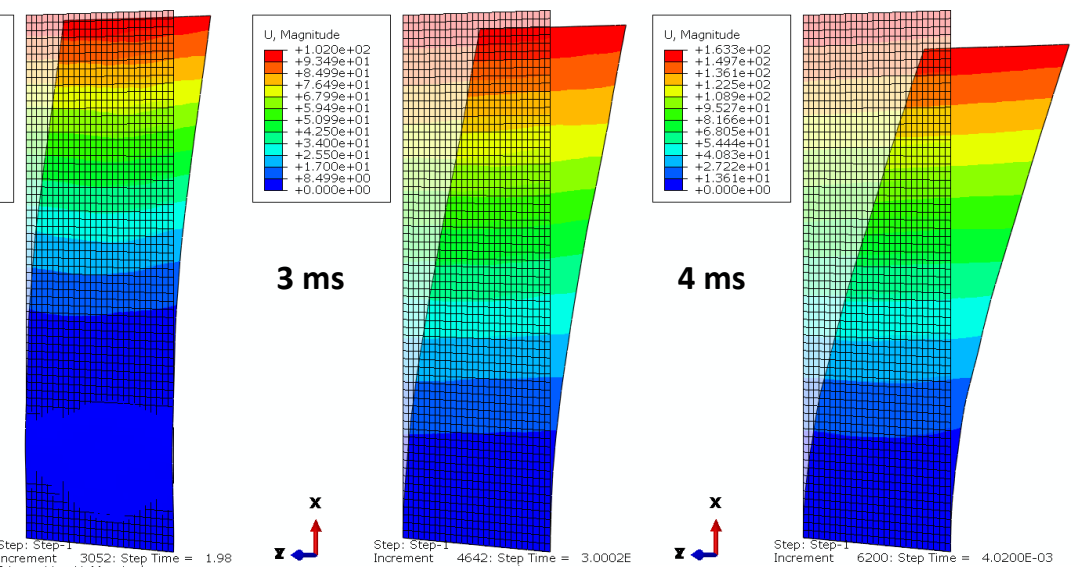

Fig. 7. Deformation series of the $90 \%$ height case

Fig. 8 gives the deformation series of the $30 \%$ height case, i.e. impacted on the location near the blade root. It can be seen that the plate deformed at the contact region in the beginning; and then maximum deflection gradually extended to the blade tip in the process of impact. In particular, the blade tip experienced a behind shifting due to the inertia force. 

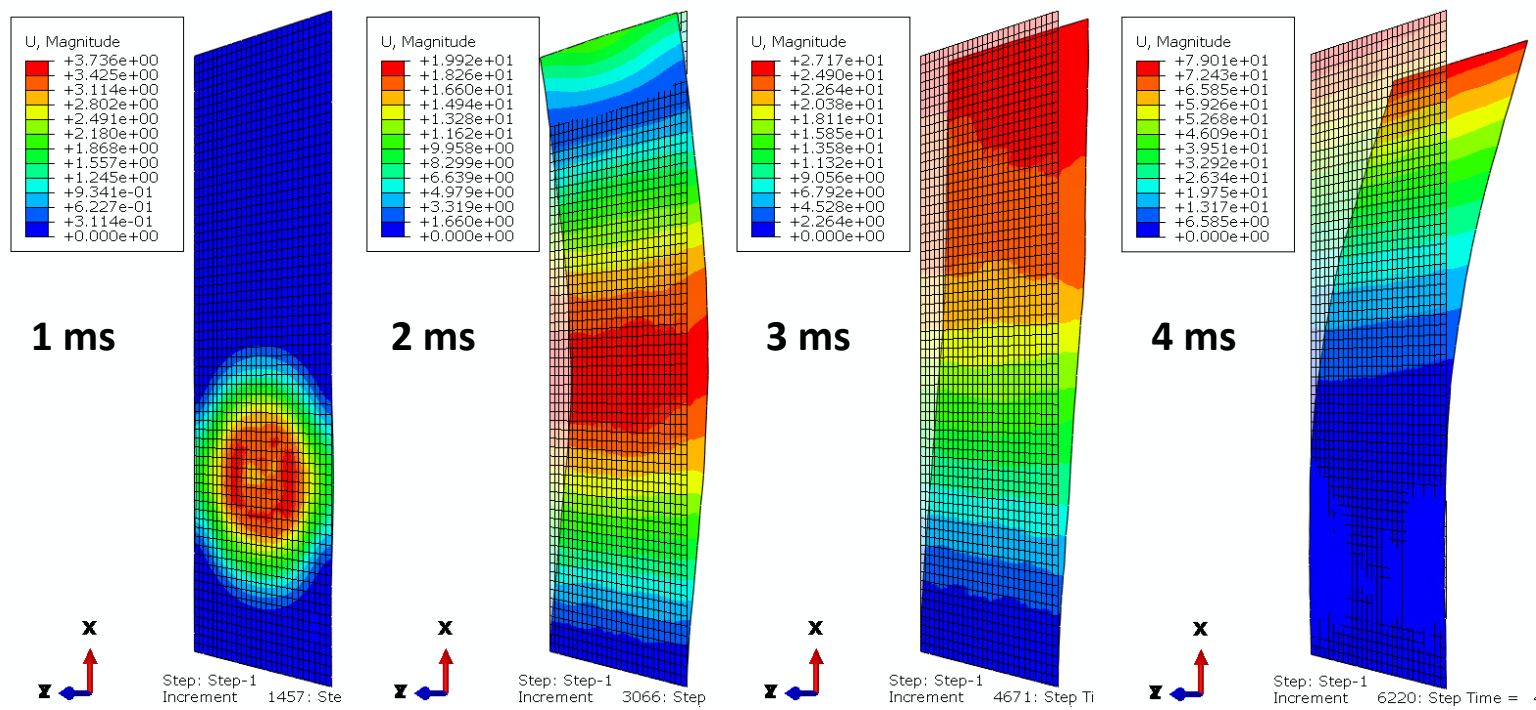

Fig. 8. Deformation series of the $30 \%$ height case

Fig. 9 shows the deformation series of the eccentrically-impacted $70 \%$ height case. Because the impact loading was not symmetric, the torsion-bending coupling deformations appeared in this case. From Figs. 6-9, it can be observed that the impact location has a significant influence on the deformation mode of the flat blades. Impacted at different locations, the bending as simply-supported beam, the bending of cantilever beam, the bending with behind shifting, and the bending-torsion coupling deformation modes appeared respectively.
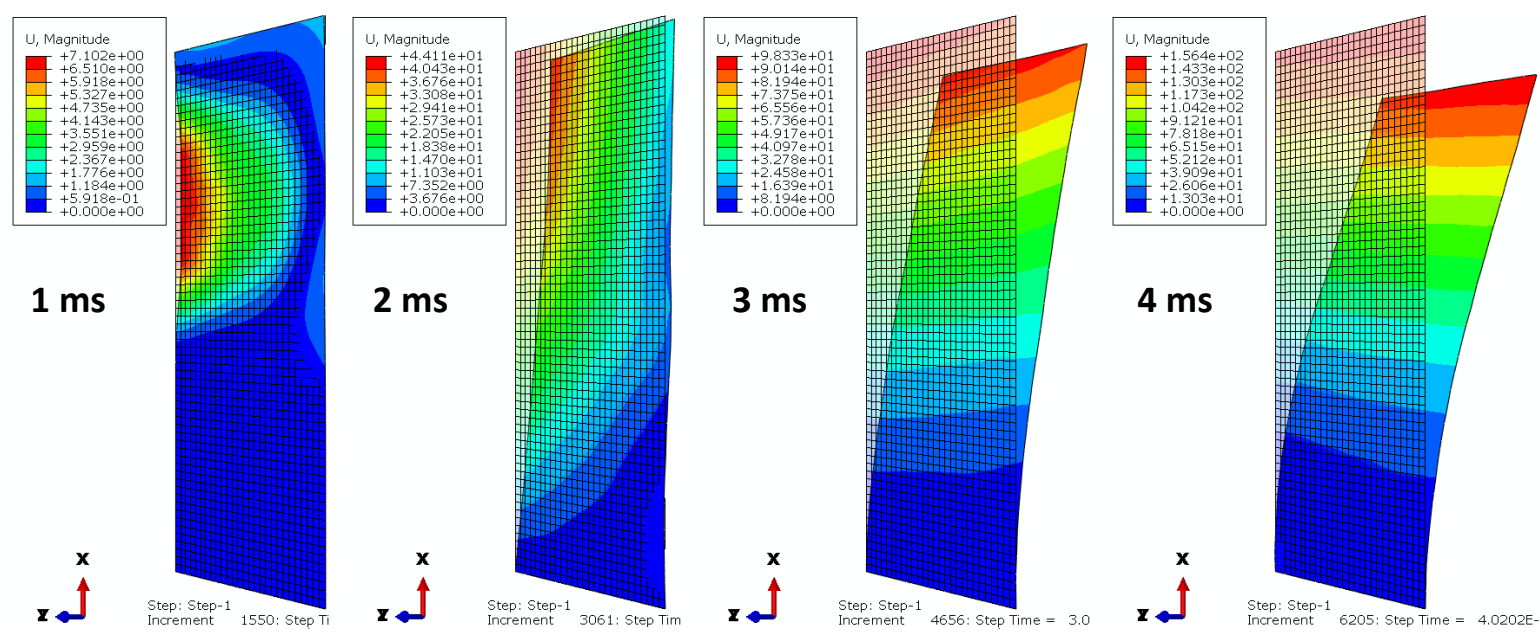

Fig. 9. Deformation series of the eccentrically-impacted $70 \%$ height case

The second considered strategy was to use an additional mass. The non-structural mass was placed in the middle of the blade tip. At beginning, a mass point with $1 \mathrm{~kg}$ was used. The results of deformation series of the $50 \%$ height with $1 \mathrm{~kg}$ point mass are shown in Fig. 10. The displacement responses are rather similar with the case without point mass. Hence, the point mass was then increased to $4 \mathrm{~kg}$. The deformation series of the $50 \%$ height with $4 \mathrm{~kg}$ point mass are shown in Fig. 11. The displacement responses were almost no difference with the case with $1 \mathrm{~kg}$ point mass. Therefore, it can be seen that additional mass has little effect on the impact deformation mode. 


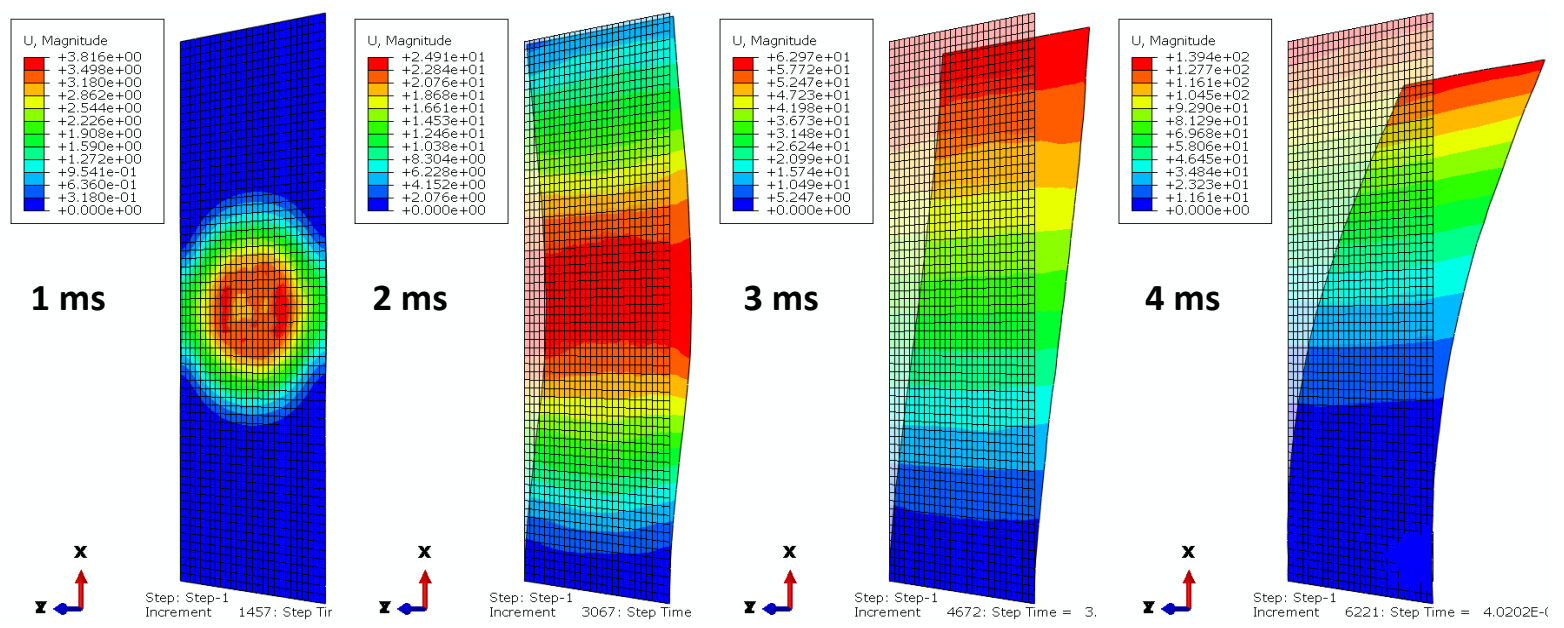

Fig. 10. Deformation series of the $50 \%$ height with $1 \mathrm{~kg}$ point mass

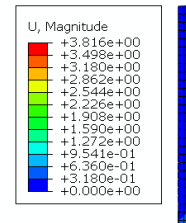

$1 \mathrm{~ms}$

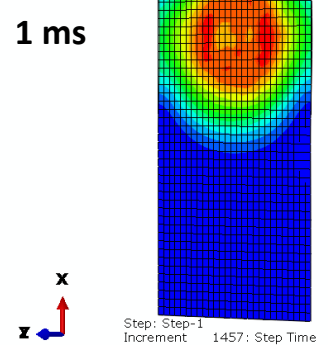

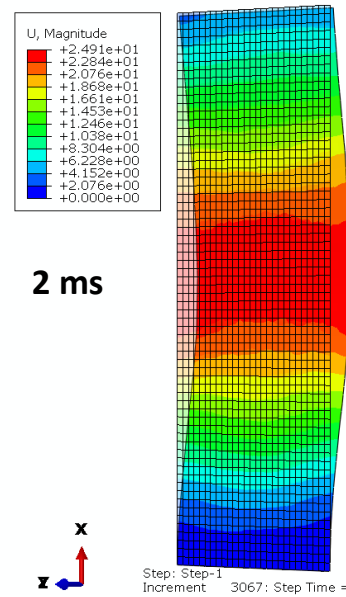
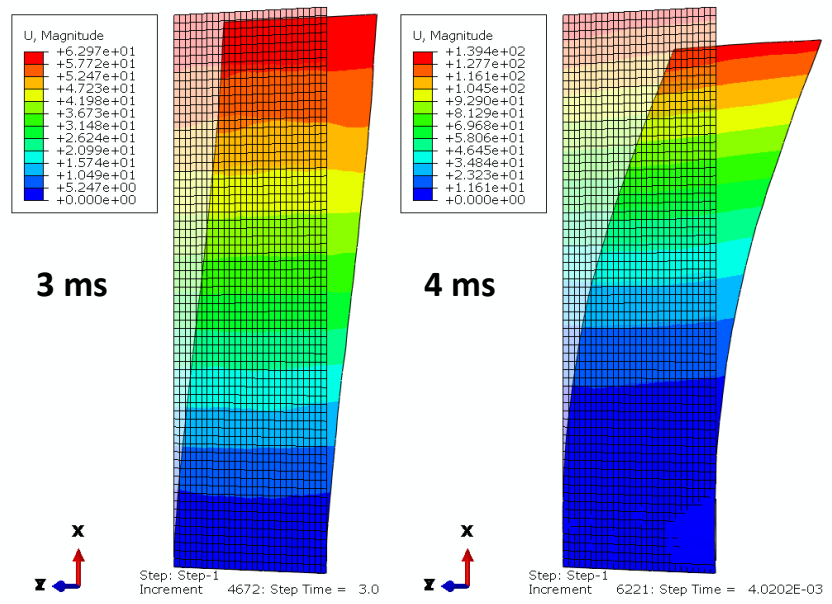

Fig. 11. Deformation series of the $50 \%$ height with $4 \mathrm{~kg}$ point mass

The third considered strategy was to change the boundary condition. A clamped-clamped boundary condition was applied. Fig. 12 shows the deformation series of the $50 \%$ height with clamped-clamped boundary condition. It can be seen the displacement responses were largely changed. The maximum deformation distributed in the middle of the blade throughout the impact process. The plate experienced bending vibrations with high frequencies. In brief, the effect of boundary conditions on the impact responses is very remarkable.
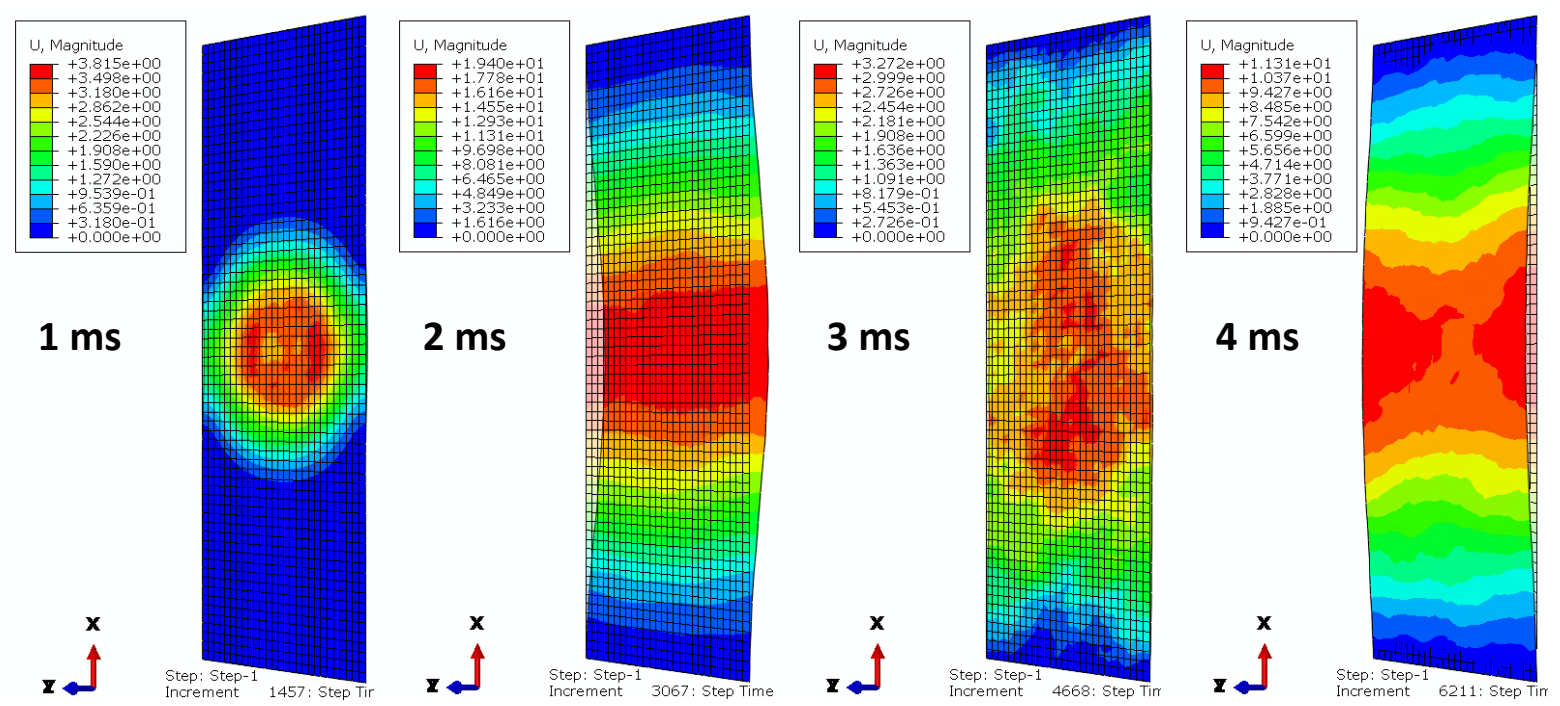

Fig. 12. Deformation series of the $50 \%$ height with clamped-clamped boundary condition 


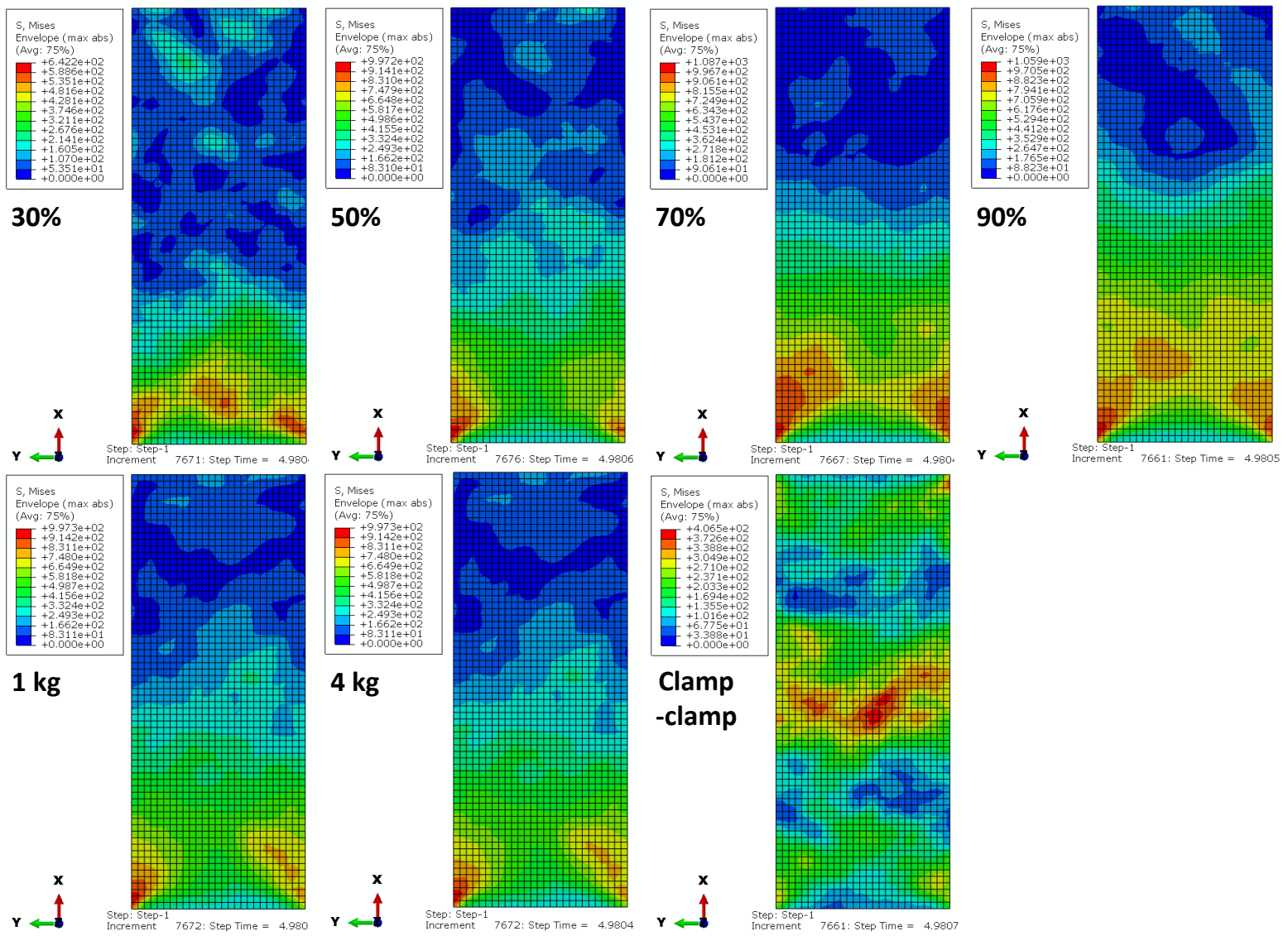

Fig. 13. The von Mises stress distributions of the above-mentioned seven blades at $5 \mathrm{~ms}$

Different displacement responses generate different stress distributions, and the latter is straightforward related to impact failure. The final goal is to obtain desirable stress distributions, which are convenient for damage mechanism study. Fig. 13 gives the von Mises stress distributions of the above-mentioned blades at $5 \mathrm{~ms}$. It can be seen that the impact location can influence the stress distributions, but the maximum stresses are all located at the blade roots for the cantilever cases. And then, additional mass has little effect on the stress responses. At last, the maximum stress of the clamped-clamped plate is distributed at the middle of the blade, and the peak value is smaller than those of any other cases. Thus, the clamped-clamped plate can be considered as a preferable surrogate case for impact testing of real fan blades.

Furthermore, in order to observe the dynamic process more completely, the time histories of the maximum deflections are compared. The maximum deflections locate at the blade tips for the cantilever cases, while locates at the plate centre for the clamped-clamped case. Fig. 14 plots the computation results. On the whole, the deflections increase as the impact locations move from the root to the tip. Then, it can be seen that the point mass has no effect on the deflection histories. In particular, the deflection history curve of clamped-clamped case exhibits a harmonic oscillation around the initial position, which is totally different from those of the cantilever ones. These results can provide a reference for both moderate deflection and stress responses in future simplified plate-level experiments. 


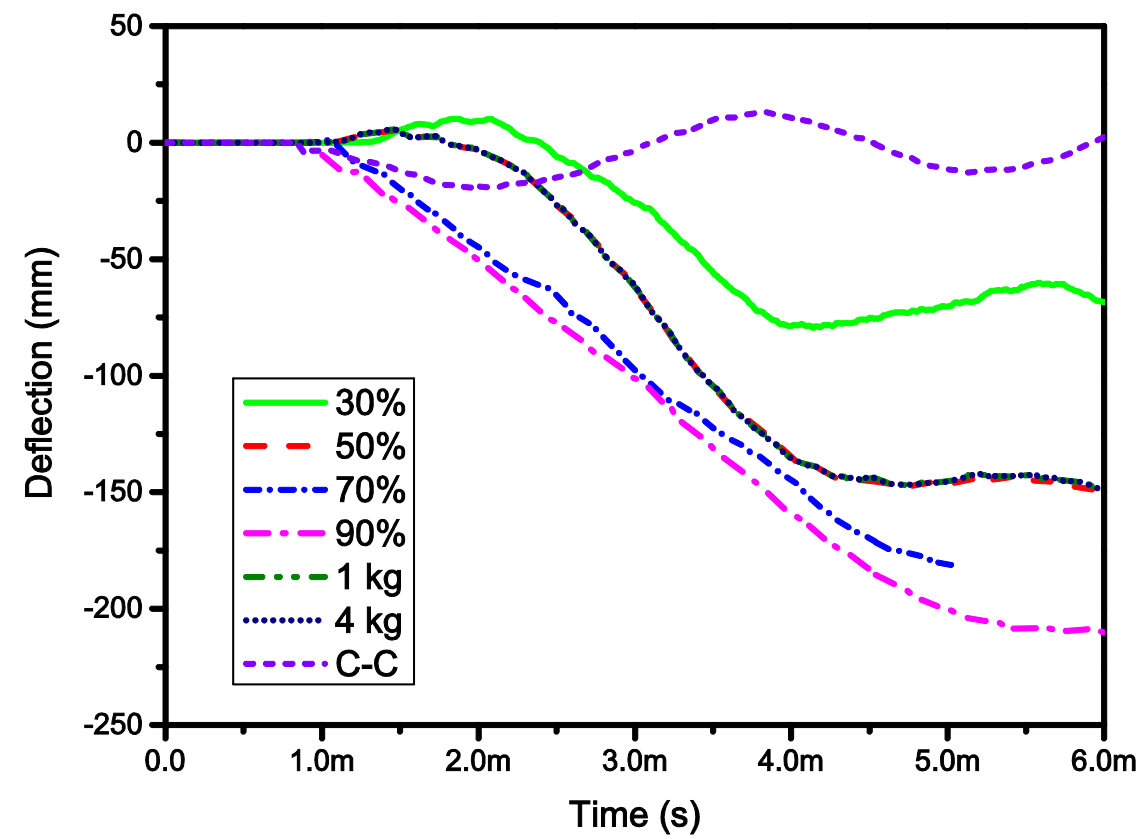

Fig. 14. Time histories of the maximum deflections in the seven considered models (C-C: clamped-clamped boundary condition)

\section{Conclusions}

To pursue desirable stress distributions of the simplified slender composite plate to mimic the real fan blades, SPHFEM simulations were conducted to examine the effect of three different strategies on the high-velocity impact responses. The following conclusions can be reached:

1. The impact location has a significant influence on the deformation mode of the flat blades. Impacted at different locations, the bending as simply-supported beam, the bending of cantilever beam, the bending with behind shifting, and the bending-torsion coupling deformation modes appeared respectively. The impact location can also influence the stress distributions, but the maximum stresses are all located at the blade roots in the cantilever cases;

2. The additional mass has little effect on the impact deformation mode, the deflection histories and the impact stress response, even though the additional weight is heavier than the plate;

3. The effect of boundary conditions on bird-strike impact responses of blade-like plates is very remarkable. Both the maximum deformation and the maximum stress of the clamped-clamped plate are distributed at the middle of the blade, and the peak value is smaller than those of any other cases, which can successfully avoid the root failure.

Therefore, in the future simplified experimental work, different boundary conditions (e.g. the clamped-clamped one in present simulation) will be the promising configurations for damage mechanism study, which can avoid the undesirable failure mode at the blade root.

\section{Data Availability}

The authors are willing to share the data underlying the findings of this work, and the data used to support the findings of this study are included within the article.

\section{Acknowledgments}

This work was jointly supported by the National Science and Technology Major Project (2017-VIII-0003-0114); the National Natural Science Foundation and the Civil Aviation Administration of China (U1333119); the Fundamental Research Fund for the Central Universities (56XBC18206, 56XBA18201, IEA180017A19). 
Author's Contributions: Conceptualization, Y Zhou; Data curation, Y Zhou; Formal analysis, Y Zhou; Investigation, Y Zhou; Methodology, Y Zhou; Resources, Y Sun; Software, Y Zhou; Supervision, Y Sun; Validation, T Huang; Visualization, Y Zhou; Writing - original draft, Y Zhou; Writing - review \& editing, Y Zhou.

Editor: Marcílio Alves.

\section{References}

Abrate, Serge. (2016). Soft impacts on aerospace structures. Progress in Aerospace Sciences, 81, 1-17. doi: 10.1016/j.paerosci.2015.11.005

Amoo, Leye M. (2013). On the design and structural analysis of jet engine fan blade structures. Progress in Aerospace Sciences, 60, 1-11. doi: 10.1016/j.paerosci.2012.08.002

Di Caprio, F., Cristillo, D., Saputo, S., Guida, M., \& Riccio, A. (2019). Crashworthiness of wing leading edges under bird impact event. Composite Structures, 216, 39-52. doi: 10.1016/j.compstruct.2019.02.069

The GEnx Commercial Aircraft Engine.). Retrieved 9/17, 2019, from https://www.geaviation.com/commercial/engines/genxengine

Guida, M., Marulo, F., Meo, M., \& Russo, S. (2012). Experimental Tests Analysis of Fiber Metal Laminate under Birdstrike. Mechanics of Advanced Materials and Structures, 19(5), 376-395. doi: 10.1080/15376494.2010.542273

Guida, M., Sellitto, A., Marulo, F., \& Riccio, A. (2019). Analysis of the Impact Dynamics of Shape Memory Alloy Hybrid Composites for Advanced Applications. Materials (Basel), 12(1). doi: 10.3390/ma12010153

Guida, Michele, Marulo, Francesco, Polito, Tiziano, Meo, Michele, \& Riccio, Massimo. (2009). Design and Testing of a FiberMetal-Laminate Bird-Strike-Resistant Leading Edge. Journal of Aircraft, 46(6), 2121-2129. doi: 10.2514/1.43943

Handschuh, Katherine M., Miller, Sandi G., Sinnott, Matthew J., Kohlman, Lee W., Roberts, Gary D., Pereira, J. Michael, \& Ruggeri, Charles R. (2014). Materials, Manufacturing and Test Development of a Composite Fan Blade Leading Edge Subcomponent for Improved Impact Resistance SAMPE Conference. Seattle, WA; United States: Society for the Advancement of Materials and Process Engineering; Covina, CA, United States.

Heimbs, Sebastian. (2011). Computational methods for bird strike simulations: A review. Computers \& Structures, 89(23-24), 2093-2112. doi: 10.1016/j.compstruc.2011.08.007

Hou, J. P., \& Ruiz, C. (2007). Soft body impact on laminated composite materials. Composites Part A: Applied Science and Manufacturing, 38(2), 505-515. doi: 10.1016/j.compositesa.2006.02.018

Kim, Minki, Zammit, Anthony, Siddens, Aaron, \& Bayandor, Javid. (2011). An Extensive Crashworthiness Methodology for Advanced Propulsion Systems, Part I: Soft Impact Damage Assessment of Composite Fan Stage Assemblies. doi: 10.2514/6.2011-980

Li, D., Zhang, W., Guo, L., Xie, W., \& Jiang, X. (2019). Energy absorption of aluminum panels subjected to gelatin projectile impact. Latin American Journal of Solids and Structures, 16(7). doi: 10.1590/1679-78255604

Li, Yulong, Zhang, Yongkang, \& Xue, Pu. (2008). Study of Similarity Law for Bird Impact on Structure. Chinese Journal of Aeronautics, 21, 512-517.

Liu, Jun, Zhong, Dudu, Li, Yulong, Tang, Zhongbin, Gao, Xiaosheng, Zhang, Zhixue, \& Huang, Fuzheng. (2018a). Numerical simulation and test on damage of rotary engine blades impacted by bird. International Journal of Crashworthiness, 1-15. doi: 10.1080/13588265.2018.1452548

Liu, Lulu, Luo, Gang, Chen, Wei, Zhao, Zhenhua, \& Huang, Xiong. (2018b). Dynamic Behavior and Damage Mechanism of 3D Braided Composite Fan Blade under Bird Impact. International Journal of Aerospace Engineering, 2018, 1-16. doi: $10.1155 / 2018 / 5906078$

Mao, Ronghai, Meguid, Shaker A., \& Ng, Teng Yong. (2007). Finite Element Modeling of a Bird Striking an Engine Fan Blade. Journal of Aircraft, 44(2), 583-596. doi: 10.2514/1.24568

Mazzariol, Leonardo M., \& Alves, Marcílio. (2019a). Experimental verification of similarity laws for impacted structures made of different materials. International Journal of Impact Engineering, 133, 103364. doi: 10.1016/j.ijimpeng.2019.103364 
Mazzariol, Leonardo M., \& Alves, Marcílio. (2019b). Similarity laws of structures under impact load: Geometric and material distortion. International Journal of Mechanical Sciences, 157-158, 633-647. doi: 10.1016/j.ijmecsci.2019.05.011

Meguid, S. A., Mao, R. H., \& Ng, T. Y. (2008). FE analysis of geometry effects of an artificial bird striking an aeroengine fan blade. International Journal of Impact Engineering, 35(6), 487-498. doi: 10.1016/j.ijimpeng.2007.04.008

Mishra, R. K., Ahmed, S. I., \& Srinivasan, K. (2013). Investigation of a Bird Strike Incident of a Military Gas Turbine Engine. Journal of Failure Analysis and Prevention, 13(6), 666-672. doi: 10.1007/s11668-013-9744-8

Mohagheghian, I., Wang, Y., Zhou, J., Yu, L., Guo, X., Yan, Y., ... Dear, J. P. (2017). Deformation and damage mechanisms of laminated glass windows subjected to high velocity soft impact. International Journal of Solids and Structures, 109, 46-62. doi: 10.1016/j.ijsolstr.2017.01.006

Muir, Eric R., \& Friedmann, Peretz P. (2016). Forced and Aeroelastic Responses of Bird-Damaged Fan Blades: A Comparison and Its Implications. Journal of Aircraft, 53(2), 561-577. doi: 10.2514/1.c033424

Nishikawa, M., Hemmi, K., \& Takeda, N. (2011). Finite-element simulation for modeling composite plates subjected to softbody, high-velocity impact for application to bird-strike problem of composite fan blades. Composite Structures, 93(5), 14161423. doi: 10.1016/j.compstruct.2010.11.012

Rayavarapu, Vijaya Kumar. (2016). Failure Analysis of Rotorcraft Composite End Plate Structure Under High-Velocity Bird Impact. Journal of Failure Analysis and Prevention, 16(5), 864-873. doi: 10.1007/s11668-016-0165-3

Roberts, Gary D, Revilock, Duane M, Binienda, Wieslaw K, Nie, Walter Z, Mackenzie, S Ben, \& Todd, Kevin B. (2002). Impact Testing and Analysis of Composites for Aircraft Engine Fan Cases. Journal of Aerospace Engineering, 15(3), 104-110. doi: 10.1061//asce/0893-1321/2002/15:3/104

Sinha, Sunil K. (2014). Transient Response of a Multilayered Composite Rotating Airfoil Under Slicing-Impact Loading. AlAA Journal, 52(12), 2701-2711. doi: 10.2514/1.j052888

Sinha, Sunil K., Turner, Kevin E., \& Jain, Nitesh. (2011). Dynamic Loading on Turbofan Blades Due to Bird-Strike. Journal of Engineering for Gas Turbines and Power, 133(12). doi: 10.1115/1.4004126

Teichman, H. C., \& Tadros, R. N. (1991). Analytical and experimental simulation of fan blade behavior and damage under bird impact. Journal of Engineering for Gas Turbines and Power-Transactions of the Asme, 113(4), 582-594.

Vignjevic, Rade, Orłowski, Michał, De Vuyst, Tom, \& Campbell, James C. (2013). A parametric study of bird strike on engine blades. International Journal of Impact Engineering, 60, 44-57. doi: 10.1016/j.ijimpeng.2013.04.003

Yu, Zeliang, Xue, Pu, Yao, Peilun, \& Zahran, M. S. (2019). Analytical determination of the critical impact location for wing leading edge under birdstrike. Latin American Journal of Solids and Structures, 16(1). doi: 10.1590/1679-78255352

Zhang, Dahai, \& Fei, Qingguo. (2016). Effect of bird geometry and impact orientation in bird striking on a rotary jet-engine fan analysis using SPH method. Aerospace Science and Technology, 54, 320-329. doi: 10.1016/j.ast.2016.05.003

Zhang, Zhuo, Li, Liang, \& Zhang, Dingguo. (2018). Effect of arbitrary yaw/pitch angle in bird strike numerical simulation using SPH method. Aerospace Science and Technology, 81, 284-293. doi: 10.1016/j.ast.2018.08.010

Zhou, Yadong, Sun, Youchao, \& Cai, Wenchao. (2019). Bird-striking damage of rotating laminates using SPH-CDM method. Aerospace Science and Technology, 84, 265-272. doi: 10.1016/j.ast.2018.10.009 